\title{
New approaches in ankylosing spondylitis
}

\section{There have been marked improvements in treatment options but none have yet been shown to induce remission}

$\mathrm{T}$

he past decade has seen major advances in the diagnosis and management of ankylosing spondylitis (AS) and in research into its pathogenesis. It remains the case that no current treatments have been shown to lead to disease remissions or to halt the progression of the bony ankylosis that causes the major morbidity associated with this condition. Nonetheless, improved diagnostic methods and management have led to major benefits for patients, with marked improvements in quality of life with reduced treatment-associated side effects.

\section{Early diagnosis and non-radiographic axial spondyloarthritis}

AS is diagnosed using the modified New York classification criteria for the disease, ${ }^{1}$ which are highly specific for AS but require the presence of $x$-ray changes in the sacroiliac joints to establish a diagnosis. Consequently, they lack sensitivity, particularly early in disease. It is estimated that it takes on average a decade between onset of axial spondyloarthritis symptoms before these $x$-ray changes develop, although in some cases more rapid progression occurs. ${ }^{2}$

The introduction of magnetic resonance imaging (MRI) scanning for the diagnosis of axial spondyloarthritis has greatly improved the ability to diagnose patients with less severe $x$-ray changes and with disease of shorter duration. This has highlighted the long pre-radiographic phase that is universal in AS, and has led to the development of new classification criteria that do not require $\mathrm{x}$-ray changes to be present. ${ }^{3}$ This new clinical entity is called non-radiographic axial spondyloarthritis (nr-axSpA) and is intended to refer to patients with inflammatory axial arthritis without $\mathrm{x}$-ray changes typical of AS. Roughly half of patients presenting with nr-axSpA will, over the space of a decade, progress to develop AS. ${ }^{4}$ At this point there is only limited capacity to predict which patients will progress or not, with some but not all studies suggesting increased likelihood of progression with male sex, HLA-B27 carriage, smoking, higher baseline C-reactive protein (CRP) levels, extent of MRI evidence of inflammation and younger age of onset. ${ }^{5}$ Whether or not such patients ultimately develop AS, they

Matt Brown Linda A Bradbury

Institute of Health and Biomedical Innovation Queensland University of Technology, Brisbane, QLD.

matt.brown@ qut.edu.au suffer significantly with their disease, with a similar disease burden to established AS, and therefore need treatment.

\section{Current management approaches}

In common with most significant chronic diseases, management of AS is best performed by multidisciplinary teams, including not only medical practitioners but also allied health professionals. In the case of AS, there are

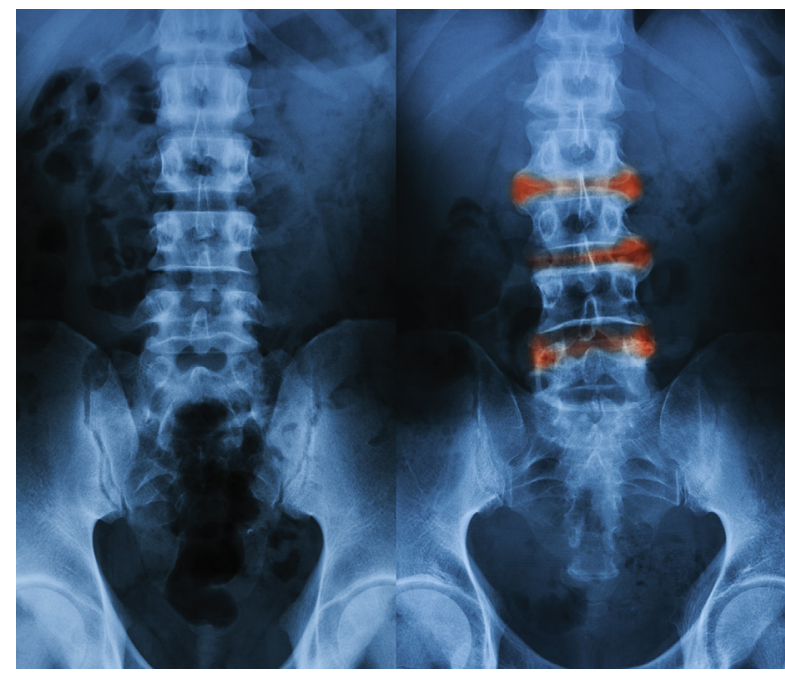

particular roles for specialist rheumatology nurses, nurse practitioners and physiotherapists.

Exercise is paramount in the management of AS and the role of the physiotherapist in guiding and supporting patients is key. ${ }^{6}$ Individualised assessment, treatment and monitoring are essential to aid adherence to a lifelong exercise program. ${ }^{7,8}$ Extended-scope physiotherapists who have undergone further training in AS may also play a role in management. ${ }^{9}$

Registered nurses with specific training in rheumatology can provide care that includes monitoring of disease activity, impact on activities of daily living, psychosocial health, drug treatment and side effects. ${ }^{10}$ Nurse practitioners can also autonomously prescribe medications within their scope of practice, refer patients to other health professionals and perform examinations and procedures such as joint aspiration and injection (http://www.health.gov.au/internet/main/publishing. nsf/Content/ work-nurse-prac). ${ }^{11}$ They also play a critical role in education about medication-related issues such as benefits and risks, management of complications, and drug administration. This is particularly important in maintaining patients safely on biological agents which are complex to administer and have potentially severe side effects, yet also bring great benefits when used appropriately.

Patient education is key to improving coping strategies and increasing self-care abilities, ${ }^{10}$ thereby achieving a greater sense of empowerment and, in turn, function. Further, education can address comorbidities such as cardiovascular disease, as well as general health issues such as smoking, diet and reinforcing the importance of exercise. Given the major effect of cigarette smoking as a risk factor for AS, and its association with increased disease activity, rate of spinal fusion and resistance to 
therapy, smoking cessation is one of the key management challenges in AS, and nurse practitioners are particularly effective in implementing such lifestyle modification programs. It has also been recognised that greater levels of knowledge were found in patients monitored by a nurse compared with those monitored by doctors. ${ }^{10}$

\section{Medical treatment}

Non-steroidal anti-inflammatory drug (NSAID) therapy is more efficacious in AS-associated pain than for pain resulting from non-inflammatory causes, and thus most patients receive these agents. It is unclear at this point whether NSAID therapy retards progression of ankylosis in AS, but it is clear that any beneficial effect is modest and probably restricted to patients with ongoing inflammation as assessed by elevation of erythrocyte sedimentation rate (ESR) or CRP levels. Use of long-acting NSAIDs, particularly when given with an evening meal, is particularly effective at controlling morning symptoms and in assisting sleep, which is frequently disturbed in patients with AS due to pain and stiffness. In the $5-10 \%$ of patients who have coexistent inflammatory bowel disease, NSAIDs may cause flares of colitis. In this setting the NSAID etoricoxib, which unlike other NSAIDs does not exacerbate inflammatory bowel disease, may be useful. ${ }^{12}$

Other disease-modifying antirheumatic drugs that are effective in rheumatoid arthritis have little or no role to play in treating AS. ${ }^{13,14}$ Sulfasalazine may be effective in AS-peripheral arthritis (knees and below or upper limb), but its beneficial effects overall are modest. ${ }^{15}$ Hip disease is considered as a component of axial disease in AS.

No trial evidence to support the use of disease-modifying antirheumatic drugs alone for hip disease has been reported to date, and therefore it cannot be recommended, as these therapies are undoubtedly associated with significant side effects. Hip inflammation in AS does respond to tumour necrosis factor inhibitor (TNFi) therapy. Sulfasalazine may also be useful in recurrent acute anterior uveitis, ${ }^{16}$ a condition that ultimately affects roughly $60 \%$ of patients. Methotrexate and sulfasalazine have no demonstrated efficacy in spinal or sacroiliac disease in AS and therefore should not be used for this indication.

Corticosteroid agents should be used with great caution. Compared with rheumatoid arthritis, AS is less responsive to oral or systemic corticosteroids. Further, AS is frequently complicated by osteoporosis, which combined with the increased spinal stiffness caused by ankylosis, leads to a significantly increased risk of spinal fracture. This spinal fragility is exacerbated by corticosteroids, further encouraging caution in their use. Intra-articular corticosteroids may be useful as a short term therapy for sacroiliitis or peripheral arthritis.

\section{Treatment of non-radiographic axial spondyloarthritis}

Clinical trials have confirmed that TNFi therapy, the gold standard treatment for established AS, is also effective in treating nr-axSpA. ${ }^{17}$ This is particularly true for cases that are of more recent onset $(<3$ years symptom duration), with elevated ESR or CRP levels, or a positive MRI scan, providing objective evidence of inflammation. In such cases, the treatment response is similar to that seen with established AS, where TNFi treatment is highly effective. Despite this evidence, no biological medication has yet been funded for use in nr-axSpA, leaving a significant group of patients for whom there is no funded therapy available. Currently the Australian Pharmaceutical Benefits Scheme (PBS) restrictions for access to TNFi for AS include that the patient meets the modified New York criteria for AS, has failed to respond to NSAID treatment and a 3-month exercise program, has high self-reported disease activity (Bath Ankylosing Spondylitis Disease Activity Index $>4$ ), and has high acute phase reactants (CRP $>10 \mathrm{mg} / \mathrm{L}, \mathrm{ESR}>25 \mathrm{~mm} / \mathrm{h})$.

A key question now is whether early treatment can lead to better long term outcomes for patients. ${ }^{18}$ There is now strong evidence to support this being the case in terms of reducing progression of ankylosis. While short term studies (up to 2 years of treatment) failed to show an effect of TNFi treatment on progression of x-ray changes, ${ }^{19}$ longer studies (4 years of treatment) have shown significant benefits, roughly halving $\mathrm{x}$-ray progression. ${ }^{20}$ This benefit is greater the earlier TNFi treatment is initiated. Whether TNFi retards progression of nr-axSpA to AS is not yet clear, although there is suggestive evidence that successful suppression of inflammation can lead to treatment-free disease remissions.

\section{New therapies}

Genetic discoveries early in the genome-wide association study era demonstrated that genetic variants in the interleukin (IL)-23 receptor pathway were strongly associated with $\mathrm{AS}^{21}$ and the importance of the pathway has been confirmed by immunological studies in humans and animal models. This led to the repositioning of drugs targeting IL-23 and the related cytokine IL-17 for trial in AS.

These trials have been highly successful, with response rates at least equivalent to those of TNFi treatment, and with the medications being well tolerated. ${ }^{22}$ Secukinumab, an IL-17 inhibitor, has therefore recently been PBS-funded in Australia for AS, with the same indications as for TNFi. These agents have been found to be more effective than TNFi for treatment of psoriasis, which complicates $5-10 \%$ of AS cases, ${ }^{23}$ but have variable effects on inflammatory bowel disease, which complicates a similar proportion of AS cases.

Up to $60 \%$ of patients with AS will, over their lifetime, experience acute anterior uveitis; ${ }^{24}$ and while this is typically easily treated with topical steroids and mydriatics, in a significant subset of patients it is recurrent or chronic and can lead to glaucoma and visual impairment. TNFi treatment is effective in reducing the frequency of acute anterior uveitis, although etanercept is less effective than the other agents in this regard. ${ }^{25}$ Whether IL-23 pathway inhibitors are effective in this condition is unknown. The effect of IL-23 pathway blockade on rate of ankylosis is also unknown.

This increasingly diverse pharmacological armamentarium opens the possibility of personalised 
approaches to medical management of patients with AS, with optimal treatment varying depending on the particular disease features that a patient may experience. Head-to-head studies will be required to determine the relative efficacy of the new agents, but given that a significant proportion of patients, for reasons related to efficacy or toxicity, cannot be managed by TNFi, the availability of alternative agents is a welcome relief for them and their caring physicians.

Despite these advances, management of AS lags behind that of the other major common immune-mediated arthropathy, rheumatoid arthritis, with fewer therapeutic options, no treatment yet convincingly shown to induce disease remission, and with less impact on slowing disease progression. This probably relates to the disproportionately low research attention AS receives in the academic sector and pharmaceutical industry relative to the burden of disease the condition causes. Given the major advances in basic research in AS, including in genetics, immunology and metagenomics, it is to be hoped that sufficient resources are made available to translate these advances into benefits for patients.

Competing interests: No relevant disclosures.

Provenance: Commissioned; externally peer reviewed.

(c) 2017 AMPCo Pty Ltd. Produced with Elsevier B.V. All rights reserved.

References are available online at www.mja.com.au. 
1 van der Linden S, Valkenburg HA, Cats A. Evaluation of diagnostic criteria for ankylosing spondylitis. A proposal for modification of the New York criteria. Arthritis Rheum 1984; 27: 361-368.

2 Reed MD, Dharmage S, Boers A, et al. Ankylosing spondylitis: an Australian experience. Intern Med J 2008; 38: 321-327.

3 Rudwaleit M, Landewe R, van der Heijde D, et al. The development of Assessment of SpondyloArthritis international Society classification criteria for axial spondyloarthritis (part I): classification of paper patients by expert opinion including uncertainty appraisal. Ann Rheum Dis 2009; 68: 770-776.

4 Mau W, Zeidler $\mathrm{H}$, Mau R, et al. Clinical features and prognosis of patients with possible ankylosing spondylitis. Results of a 10-year followup. J Rheumatol 1988; 15:1109-1114.

5 Sieper J, Rudwaleit M, Lenaerts J, et al. Partial remission in ankylosing spondylitis and non-radiographic axial spondyloarthritis in treatment with infliximab plus naproxen or naproxen alone: associations between partial remission and baseline disease characteristics. Rheumatology (Oxford) 2016; 55: 1946-1953.

6 Millner JR, Barron JS, Beinke KM, et al. Exercise for ankylosing spondylitis: an evidence-based consensus statement. Semin Arthritis Rheum 2016; 45: $411-427$.

7 O'Dwyer T, McGowan E, O'Shea F, Wilson F. Physical activity and exercise: perspectives of adults with ankylosing spondylitis. J Phys Act Health 2016; 13: 504-513.

8 Arturi $\mathrm{P}$, Schneeberger EE, Sommerfleck F, et al. Adherence to treatment in patients with ankylosing spondylitis. Clin Rheumatol 2013; 32: 1007-1015.

9 Van Rossen L, Withrington RH. Improving the standard of care for people with ankylosing spondylitis and a new approach to developing specialist ESP-Led AS clinics. Musculoskeletal Care 2012; 10: 171-177.

10 van Eijk-Hustings $Y$, van Tubergen A, Bostrom C, et al. EULAR recommendations for the role of the nurse in the management of chronic inflammatory arthritis. Ann Rheum Dis 2012; 71: 13-19.

11 Lesko M, Young M, Higham R. Managing inflammatory arthritides: role of the nurse practitioner and physician assistant. J Am Acad Nurse Pract 2010; 22: 382-392.

12 El Miedany Y, Youssef S, Ahmed I, El Gaafary M. The gastrointestinal safety and effect on disease activity of etoricoxib, a selective cox-2 inhibitor in inflammatory bowel diseases. Am J Gastroenterol 2006; 101: 311-317.

13 Ward MM, Deodhar A, Akl EA, et al. American College of Rheumatology/ Spondylitis Association of America/Spondyloarthritis Research and Treatment Network 2015 recommendations for the treatment of ankylosing spondylitis and nonradiographic axial spondyloarthritis. Arthritis Rheumatol 2016; 68: 282-298.

14 Robinson PC, Bird P, Lim I, et al. Consensus statement on the investigation and management of non-radiographic axial spondyloarthritis (nr-axSpA). Int J Rheum Dis 2014; 17: 548-556.

15 Braun J, Pavelka K, Ramos-Remus C, et al. Clinical efficacy of etanercept versus sulfasalazine in ankylosing spondylitis subjects with peripheral joint involvement. J Rheumatol 2012; 39: 836-840.

16 Benitez-Del-Castillo JM, Garcia-Sanchez J, Iradier T, Banares A. Sulfasalazine in the prevention of anterior uveitis associated with ankylosing spondylitis. Eye (Lond) 2000; 14 (Pt 3A): 340-343.

17 Sieper J, van der Heijde D, Dougados M, et al. Efficacy and safety of adalimumab in patients with non-radiographic axial spondyloarthritis: results of a randomised placebo-controlled trial (ABILITY-1). Ann Rheum Dis 2013; 72: 815-822

18 Robinson PC, Brown MA. The window of opportunity: a relevant concept for axial spondyloarthritis. Arthritis Res Ther 2014; 16: 109.

19 van der Heijde D, Salonen D, Weissman BN, et al. Assessment of radiographic progression in the spines of patients with ankylosing spondylitis treated with adalimumab for up to 2 years. Arthritis Res Ther 2009; 11: R127.

20 Haroon N, Inman RD, Learch TJ, et al. The impact of tumor necrosis factor alpha inhibitors on radiographic progression in ankylosing spondylitis. Arthritis Rheum 2013; 65: 2645-2654.

21 Wellcome Trust Case Control Consortium, Australo-Anglo-American Spondylitis Consortium, Burton PR, et al. Association scan of 14,500 nonsynonymous SNPs in four diseases identifies autoimmunity variants. Nat Genet 2007; 39: 1329-1337.

22 Sieper J, Deodhar A, Marzo-Ortega $\mathrm{H}$, et al. Secukinumab efficacy in anti-TNF-naive and anti-TNF-experienced subjects with active ankylosing spondylitis: results from the MEASURE 2 Study. Ann Rheum Dis 2016. doi:10.1136/annrheumdis-2016-210023

23 Griffiths CE, Reich K, Lebwohl M, et al. Comparison of ixekizumab with etanercept or placebo in moderate-to-severe psoriasis (UNCOVER-2 and UNCOVER-3): results from two phase 3 randomised trials. Lancet 2015; 386: 541-551.

24 Robinson PC. The genetics of ankylosing spondylitis and anterior uveitis [PhD thesis]. Brisbane: University of Queensland, 2014

25 Fabiani C, Vitale A, Lopalco G, et al. Different roles of TNF inhibitors in acute anterior uveitis associated with ankylosing spondylitis: state of the art. Clin Rheumatol 2016; 35: 2631-2638. 